\title{
DECIDIR EN MODO JUSTIFICADO: UNA EXPLICACIÓN COHERENTISTA
}

María Cristina Redondo*

* Conicet
El trabajo de Amalia Amaya, tal como ella misma lo presenta, es "acerca de la naturaleza de la coherencia y su papel en el razonamiento jurídico". Aunque ésta es una descripción adecuada, cabe agregar que su propuesta incluye un conjunto nutrido de ideas muy concretas. Sobre la base de razones bien detalladas Amaya defiende una serie de tesis sobre cómo han de entenderse la coherencia y la toma de decisiones justificadas, en general, pero también ofrece una específica teoría, ciertamente de tipo coherentista, acerca de la justificación de las decisiones judiciales en particular. Se trata de una excelente presentación que aborda múltiples temas, pone en relación el enfoque coherentista de la justificación con otras perspectivas y 
ofrece buenos argumentos a favor de ideas muy atractivas. En este sentido, la riqueza del trabajo abre numerosos frentes de discusión en los que no soy competente para opinar, pero al mismo tiempo da lugar a muchas preguntas y reflexiones que sí me siento legitimada a expresar. A continuación voy a tratar de exponer algunas de estas preguntas y reflexiones.

\section{I}

Doy por descontado que Amaya defiende en general una teoría epistemológica de tipo coherentista y que, en este caso, la aplica a la actividad judicial. Específicamente, la aplica a aquella actividad mediante la cual los jueces responden a la quaestio iuris y a la quaestio facti cuando dictan sentencia. Entiendo también que su propuesta teórica es de tipo tanto explicativo como normativo. Es decir, por una parte, pretende dar cuenta del modo en que los jueces efectivamente razonan $\mathrm{y}$ arriban a sus conclusiones. Por otra parte, ofrece un modelo normativo que permite evaluar si, y cuándo, las decisiones de los jueces pueden considerarse justificadas.

Teniendo esto presente, una primera cuestión sobre la que me interesa reflexionar es si una teoría epistemológica de este tipo en realidad compite con las alternativas con las que Amaya la confronta en el trabajo, y en comparación con las cuales se encontraría en una mejor posición. Dice la autora ${ }^{1}$ :

Frente a teorías de la decisión judicial que cuentan con una clara descripción de los patrones de inferencia aceptables -i.e., la lógica deductiva- y las teorías del razonamiento acerca de hechos que emplean las sofisticadas herramientas de la lógica inductiva -i.e., el bayesianismo- el coherentismo parece estar desprovisto de una teoría sólida acerca de cómo funcionan -si es que funcionan- los argumentos de coherencia. La tesis que quisiera proponer aquí es que el coherentismo, por el contrario, sí que cuenta con una clara descripción de los

\footnotetext{
${ }^{1}$ Cf. A. Amaya, “Diez tesis acerca de la coherencia en el Derecho", apartado 2. 
procesos de inferencia que resultan en creencias justificadas. La inferencia coherentista es un tipo de inferencia explicativa $\mathrm{y}$, por lo tanto, contamos con las herramientas de la lógica abductiva para dar cuenta del tipo de inferencias que, según el coherentismo, confieren justificación.

Varias inquietudes surgen a partir de estas afirmaciones. A tenor de este punto de vista, el coherentismo, aplicado a la justificación de las decisiones judiciales, estaría en competición con otras teorías que usan la lógica deductiva e inductiva en el tentativo de ofrecer una "descripción de los procesos de inferencia que resultan en creencias justificadas". Dado que esta tesis se presta a interpretaciones equívocas conviene precisar desde el comienzo algunos puntos. Sin necesidad de entrar en el espinoso asunto relativo al status de la lógica en general, podemos afirmar pacíficamente que la aplicación de las herramientas de la lógica deductiva e inductiva en los estudios relativos a la justificación de la decisión judicial no tiene como objetivo describir, ni prescribir, los procesos seguidos, o a seguir, en la toma de dichas decisiones. Las teorías que utilizan la lógica -deductiva o inductiva- en este contexto, usan sus respectivos esquemas de inferencia como herramientas para dictaminar cuándo el contenido de una conclusión está justificado -deductiva o inductivamente-, con independencia del proceso inferencial del que tal conclusión resulte ${ }^{2}$. En otras palabras, la aplicación de la lógica a cualquier tipo de decisión, incluida la decisión judicial, no tiene por objeto el comportamiento de los agentes decisores, i.e. el proceso mediante el cual se arriba a una conclusión o se toma una decisión. La lógica deductiva y la inductiva establecen sólo las distintas formas en que un enunciado tiene que estar respaldado para que pueda afirmarse que es un enunciado justificado. Si esto es así, entonces es dudoso que el modelo de inferencia propuesto por la teoría coherentista, que según Amaya sí pretende dar cuenta del proceso a partir del cual emergen creencias justificadas, sea comparable y compita con la aplicación de los modelos deductivos e inductivos en este ámbito.

\footnotetext{
${ }^{2}$ Cf. I. Copi, Introduction to Logic, The Mac Millan Company, New York, 1953. Se cita la traducción castellana: Introducción a la lógica, Editorial Universitaria de Bs. As. 1962, pp. 17-20.
} 
Amaya entiende que el coherentismo da cuenta de un específico modelo de inferencia justificativa: la abducción. No voy a poner aquí en cuestión la tesis según la cual la abducción puede ser considerada una forma de inferencia independiente, perteneciente a un específico tipo lógico (la lógica abductiva) y, en tal sentido, autónoma respecto de las inferencias de tipo deductivo e inductivo. Sólo quisiera subrayar que, tal como la propone Amaya en este contexto, hay una importante diferencia entre una inferencia a la mejor explicación y los esquemas de la lógica deductiva o inductiva. Esta diferencia pasa desapercibida en el trabajo de Amaya porque en él se habla, sin especificar, de modelos inferenciales que permiten justificar una decisión. El problema es justamente que -si bien por distintas razonestanto la expresión "modelo inferencial" como "decisión" son ambiguas.

La diferencia a la que me refiero se pone en evidencia sólo cuando advertimos un específico tipo de ambigüedad que afecta a la expresión "decisión”. Me explico. Todas las expresiones que se refieren a estados mentales, actitudes, o acciones lingüísticas asociados a contenidos proposicionales padecen un tipo de ambigüedad, al que en este contexto podríamos llamar "ideal-empírico"3. Dichas expresiones pueden referirse tanto a un contenido proposicional como a un estado de cosas del mundo concreto (interno o externo al agente) ${ }^{4}$. Creo importante

\footnotetext{
${ }^{3}$ El problema al que me refiero puede asociarse a una advertencia de G.H. von Wright. Según este autor cabe distinguir entre estados de cosas, eventos, o sucesos genéricos e individuales. En el primer caso, se hace referencia a clases o contenidos abstractos definidos por propiedades, en el segundo caso se hace referencia a datos empíricos localizados en el espacio-tiempo. Cf. G.H. von Wright, Norma y acción. Una investigación lógica, Tecnos, Madrid, 1970. pp. 4446. En la medida en que no se especifica si este tipo de expresiones se usan en uno u otro sentido ellas padecerían el tipo de ambigüedad al que me estoy refiriendo.

${ }^{4}$ Expresiones del tipo al que me estoy refiriendo, en principio, aluden a entidades pertenecientes al mundo 3 de Popper. Por ejemplo, "mi libro favorito", "el David de Michelangelo" o "la teoría de Popper sobre la pluralidad de mundos". Sin embargo, a mi entender, son asimismo ejemplos de este tipo de expresiones aquellas que, en el lenguaje de Popper, designan entidades del mundo 2, en la medida en que ellas también se refieren tanto a un evento físico como a un significado o contenido proposicional. Por ejemplo,
} 
distinguir este tipo de ambigüedad ya que, en el caso de los nombres de específicas acciones lingüísticas (por ejemplo: "decisión", "justificación", "formulación", "prescripción", "promesa", etc.), ella se suma a la más familiar ambigüedad denominada "proceso-producto". En otras palabras, es verdad que, como señalara oportunamente R. Caracciolo, "decisión" puede hacer referencia tanto a un acto o actividad como a un resultado ${ }^{5}$. Esta distinción es fundamental y, por ejemplo, en ella está centrada la literatura sobre la paradoja o el predicamento que genera el posible contraste entre la bondad de un proceso (acto o actividad) de toma de decisión y la bondad de sus productos ${ }^{6}$. Sin embargo, cabe recordar que proceso y producto, si bien han de ser claramente diferenciados, son fases de una misma acción. En otras palabras, la acción de decidir, como cualquier otra, es un evento complejo. Consta siempre de un elemento interno, ocasionalmente compuesto de diferentes fases -es decir, la decisión como proceso- que culmina en un resultado, es decir, la decisión como producto. El problema que aquí quiero destacar es que, tratándose de acciones lingüísticas, tanto el proceso como el resultado están asociados a contenidos proposicionales. Por este motivo, las palabras que denotan estas acciones lingüísticas son ulteriormente ambiguas. Ellas no sólo hacen referencia por una parte al proceso y por otra parte al producto resultante, también refieren, por una parte, a la acción en todas sus fases (tanto al proceso como al producto) $\mathrm{y}$, por otra, a los contenidos proposicionales asociados a las diferentes fases de la acción. Como dije antes, los nombres de acciones lingüísticas (como "decisión", "justificación", "promesa", etc.) comparten este tipo de ambigüedad con las palabras que denotan actitudes proposicionales

\footnotetext{
"los recuerdos de Paolo", "la decisión de Sofía", "los deseos de mi hermano". Según Popper, las entidades referidas en este caso pertenecen a un mundo subjetivo, interno al agente. Lo que interesa destacar aquí es que se trata de expresiones que pueden referir ya sea a un contenido como a un estado de cosas físico, en este caso interno a un agente.

${ }^{5}$ Cf. R. Caracciolo, "Justificación normativa y pertenencia. Modelos de la decisión judicial” (1988), en R. Caracciolo, El Derecho desde la Filosofía, Centro de Estudios Políticos y Constitucionales, Madrid, 2009, pp. 37-69.

${ }^{6} \mathrm{Al}$ respecto, por ejemplo, véase J. L. Martí, La republica deliberativa. Una teoría de la democracia, Marcial Pons, Barcelona, 2006, pp.135-166.
} 
(como "creencia" o "deseo", "preferencia" etc.), sólo que estas últimas carecen de la ambiguiedad "proceso-producto". Esto sirve a mostrar que se trata de dos tipos de ambigüedad diferentes, y que la ambigüedad "proceso-producto" no debería confundirse con la que aquí he llamado "ideal-empírico".

Esta reflexión general está abocada a llamar la atención sobre lo siguiente. El trabajo de Amaya tiene como objetivo proponer una epistemología apropiada para el conocimiento de los hechos y del derecho en el contexto de un proceso judicial. En tal sentido, un buen método para guiar la acción de conocer y, en especial, la de justificar -tal como Amaya sostiene- podría ser un método abductivo, que siga los pasos de una inferencia a la mejor explicación. Sin embargo, los modelos relevantes en este debate acerca del método o los pasos a seguir en el desarrollo de este tipo actividad -que son los que estudia la epistemología- no compiten en absoluto con los modelos o estructuras que puede asumir la relación de implicación o de apoyo justificativo entre contenidos proposicionales - que son los que estudia la lógica. Es verdad que los modelos de inferencia que propone la lógica pueden considerarse instrumentos de justificación o que permiten controlar la justificación de una decisión, pero no de la acción de justificar o de decidir (ni de sus procesos ni de sus productos) sino de los contenidos involucrados en ellas.

Si aceptásemos que la inferencia coherentista es un tipo de inferencia lógica que compite con las distintas formas de inferencia deductivas e inductivas en el control de las decisiones judiciales, ella debería considerarse un instrumento que permite evaluar la justificación del contenido de dichas decisiones, con independencia de los procesos por los cuales se ha llegado a ellos. Sin embargo, muchos son los pasos en el trabajo de Amaya donde se sostiene que el mayor interés de la teoría coherentista -y de su modelo típico de inferencia- reside justamente en que dicha teoría da cuenta de los procesos efectivos de formación de creencias o toma de decisiones. Por supuesto, sin negar que la teoría coherentista ofrece también un modelo ideal (el modelo de proceso epistémicamente responsable) que sirve como guía sustancial de dicho proceso. En otras palabras, es claro que la teoría coherentista, con su forma típica de inferencia, sí pretende dar cuenta del proceso o 
de la acción mediante los que se decide o se justifican las decisiones, pero justamente por ello, y en tal medida, no es comparable ni compite con el aporte de la lógica a este respecto.

No es parte del objetivo de este trabajo evaluar si efectivamente la inferencia a la mejor explicación puede ser considerada una nueva forma de inferencia lógica (abductiva), diferente de las formas deductivas e inductivas. Lo que intento subrayar es que la aplicación que propone Amaya persigue una finalidad diferente de la que tiene la aplicación de las formas de inferencia deductivas e inductivas y, sobre todo, tiene un objeto diferente. Podemos aceptar que tanto la lógica como la epistemología permiten evaluar si ciertos contenidos proposicionales están justificados o no, pero lo hacen desde distintos puntos de vista. Desde un punto de vista epistemológico el interés reside en la justificación de los contenidos involucrados en un proceso real de razonamiento que, a través de distintas fases, conduce a un determinado estado de cosas resultante: una concreta creencia, o la aceptación de una específica proposición. Desde un punto de vista lógico, en cambio, el interés es totalmente abstracto y reside en la justificación de contenidos proposicionales, independientemente de que ellos sean o no objeto de las creencias de un determinado agente, o sean o no parte en un concreto proceso de inferencia.

Valiéndonos de la nota distinción trazada por J. Rawls con relación a la noción de justicia, una propuesta epistemológica puede ser interpretada como ofreciendo un modelo, no de justicia procedimental, pero sí de justificación epistémica procedimental ${ }^{7}$. Desde esta perspectiva, si dijésemos que no existen criterios para establecer la verdad de una proposición y que un agente está justificado o autorizado a creer todas y sólo aquellas proposiciones que pueden obtenerse mediante un proceso que satisface las restricciones que la teoría epistemológica impone, estaríamos entendiendo la teoría epistemológica como un modelo de justificación procedimental pura, que no presupone la existencia de criterios independientes que establezcan lo que es correcto creer. Si en cambio creemos que las proposiciones son

${ }^{7}$ Cf. J. Rawls, A Theory of Justice, Harvard University Press, Cambridge, Massachusetts, 1971, p. 85. 
verdaderas o falsas en virtud de la satisfacción de criterios independientes, pero que un razonamiento respetuoso de una propuesta epistemológica nos garantiza que siempre llegaremos a creencias verdaderas, estaríamos entendiendo esta propuesta como un modelo perfecto de justificación epistémica. Ninguna de estas lecturas parece coincidir con la opinión de Amaya quien, si entiendo bien, propone la teoría coherentista como un modelo de justificación epistémica imperfecta. Es decir, si aceptamos que la noción de verdad no es reducible a la de justificación, y aceptamos también que deberíamos creer aquello que es verdadero, el método coherentista aparece como un instrumento valioso, aunque falible, que nos guía en la búsqueda de aquello que deberíamos creer. Esta interpretación se encuentra avalada por el hecho que Amaya, si bien confía en el valor del método coherentista para acercarnos a la verdad, no cree que la creencia más coherente, i.e. aquella cuya aceptación está justificada desde una perspectiva coherentista, sea por ello una creencia verdadera ${ }^{8}$.

Sobre la base de estas consideraciones cabe concluir que, por cuanto se refiere a la justificación del conocimiento en general y de las decisiones judiciales en particular, la posibilidad de aplicar un modelo coherentista es independiente de, y no está en competición con, la aplicación de la lógica y sus formas típicas de inferencia. Concretamente, supongamos que se asume un tipo de coherentismo como modelo normativo ideal de aquello en lo que consiste una acción de decisión justificada respecto de las premisas normativas y fácticas de la decisión judicial. Esto significa que la elección de estas premisas estará justificada si, y sólo si, es "el resultado de un proceso epistémicamente responsable de maximización de la coherencia". Sin embargo, esto no significa, por ejemplo, que entre el contenido de las premisas y el contenido de la conclusión final no deba mediar una relación de deducibilidad. De hecho, el dispositivo de una sentencia

\footnotetext{
${ }^{8}$ Amaya sostiene que una de las razones fundamentales para aceptar un método de justificación coherentista es su valor epistémico. Específicamente, según la autora, "parece haber buenas razones para sostener que creencias acerca del Derecho justificadas en virtud de su coherencia son, probablemente, creencias verdaderas o correctas". Cf. A. Amaya, “Diez tesis acerca de la coherencia en el derecho", apartado 7.
} 
puede estar o no justificado, en sentido lógico, independientemente de que el acto o proceso de decisión sobre las premisas normativas y fácticas en las que tal dispositivo se apoya esté o no justificado, en el sentido epistemológico. Asimismo, podríamos abrazar una propuesta epistemológica coherentista como modelo-guía de la actividad de identificación de las premisas normativas y fácticas sin negar que, a la par, deban ofrecerse argumentos deductivos (silogismos hipotéticos, disyuntivos, reducciones al absurdo, etc.) o inductivos (inducciones en sentido estricto, inducciones estadísticas, analogías, etc.) a favor del contenido de tales premisas.

\section{II}

Cabe observar que hasta aquí he interpretado la inferencia coherentista a la que se refiere Amaya como un verdadero modelo de justificación. Modelo que constituiría el contenido de una pauta procedimental o metodológica que guía aquellos comportamientos tendientes a obtener conocimiento o a tomar decisiones justificadas. Un modo diferente de interpretar la inferencia coherentista en modo compatible y complementario con el rol que suele conferirse a las estructuras inferenciales de la lógica es entenderla como un modelo de reconstrucción conceptual. Me explico. Por ejemplo, diversas teorías sostienen que el esquema del modus ponens puede usarse para reconstruir formalmente el concepto de justificación interna de la decisión del juez o, también, el propio concepto de sentencia judicial. Es decir, entienden que la estructura del modus ponens capta un elemento definitorio de los conceptos de justificación interna, o de sentencia judicial $^{9}$. Del mismo modo, podría conjeturarse, la forma de la inferencia abductiva capta un elemento esencial de la noción de justificación externa (i.e. la justificación de la elección de las premisas normativas y

${ }^{9}$ Por ejemplo, Bulygin lo utiliza para reconstruir el concepto de sentencia judicial. Cf. E. Bulygin, "Sentencia judicial y creación de derecho”, en E. Bulygin y C. Alchourrón, Análisis Lógico y derecho, Centro de Estudios Constitucionales, Madrid, 1991, p. 357. Del mismo modo, Guastini lo usa para 
fácticas de la sentencia judicial). Bajo este presupuesto, entonces, la inferencia abductiva permitiría reconstruir la estructura profunda de este tipo de justificación. Decir que una decisión está justificada -en este sentido externo- significa que ella es el resultado de una inferencia a la mejor explicación. Consecuentemente, el análisis de una decisión externamente justificada debería mostrar que ella presenta todas las características y fases de una inferencia a la mejor explicación.

Entendida como un esquema de reconstrucción conceptual, la inferencia abductiva tampoco parece competir con las formas deductivas e inductivas de inferencia. Al menos, no existen teorías que sostengan que la decisión sobre las premisas normativas o fácticas de la sentencia deba poder ser reconstruida bajo la forma del modus ponens, u otro tipo de estructura deductiva. Asimismo, si bien la gran parte de los estudios acerca de la decisión sobre las premisas fácticas concuerdan en que tal decisión debe contar con apoyo inductivo, no por ello sostienen que, para estar externamente justificada, la decisión sobre los hechos deba poder reconstruirse bajo la forma de una determinada estructura de inferencia inductiva.

Si aceptásemos lo sugerido en los precedentes párrafos, cuando se exige que una decisión esté internamente justificada, por definición, lo que se estaría exigiendo es que ella sea un contenido susceptible de reconstruirse como conclusión de una inferencia modus ponens. Asimismo, cuando se exige que una decisión esté externamente justificada, por definición, lo que se estaría exigiendo es que la elección de las premisas en que se basa pueda reconstruirse como el resultado de una inferencia a la mejor explicación. Estos dos modelos inferenciales (el modus ponens y la inferencia a la mejor explicación) no compiten entre sí. Ellos permiten reconstruir dos conceptos diferentes de justificación: justificación interna en el primer caso, justificación externa en el segundo. Aun si creo que ésta es una lectura interesante, no veo que ella pueda ser atribuida a Amaya. Su trabajo no se propone como el análisis del concepto de justificación externa. Parece ser más fiel a la intención de la autora sostener que ella nos está proponiendo un modelo normativo de justificación epistémica.

reconstruir la noción de justificación interna. Cf. R. Guastini, L'interpretazione dei documenti normativi, Giuffrè editore, Milano, pp. 124-125. 


\section{III}

Suponiendo que esta última interpretación es correcta, enfrentamos una dificultad. En línea de principio Amaya parece sostener un modelo normativo de tipo procedimental. La exigencia de coherencia se satisface mediante el respeto de una serie de pautas (restricciones positivas y negativas) que, aplicadas al proceso de decisión, maximizarían la coherencia. Estas restricciones, que deben guiar la acción de decidir, permitirían "enunciar una serie de criterios precisos para evaluar la coherencia".

No obstante ello, paralelamente a estas pautas, Amaya enuncia un criterio general que permite evaluar cuándo un resultado está justificado, independientemente del procedimiento que se haya seguido para llegar a dicho resultado. Concretamente, su tesis es que ${ }^{10}$ : "una hipótesis acerca de los hechos o del derecho está justificada si podría ser el resultado de un proceso epistémicamente responsable de maximización de la coherencia".

Como se ve, este criterio no se refiere a cómo deben proceder los agentes en su búsqueda de las hipótesis que han de considerarse justificadas. Conforme a él, no es preciso que el contenido de la decisión resulte causalmente de un determinado proceso de razonamiento. Es imprescindible, en cambio, que el contenido de la decisión pueda ser el resultado del razonamiento de un agente epistémicamente responsable, i.e. un agente coherentista ideal y virtuoso que -y esto debemos presuponerlo- sí sigue efectivamente un procedimiento de inferencia coherentista. Continuando con la analogía antes empleada, cabría decir que Amaya paralelamente al "modelo de justificación procedimental" establecido por los principios de coherencia explicativa propone un criterio que modela un resultado: un "modelo de justificación de resultado".

En primer lugar, y en la medida en que la teoría de Amaya ofrece un criterio que permite identificar resultados justificados, ella efectivamente compite -si bien parcialmente- con otras teorías, formales

${ }^{10}$ Cf. A. Amaya, "Diez tesis acerca de la coherencia en el derecho", apartado 4. La cursiva es mía. 
o sustanciales, instrumentales o no instrumentales, que ofrecen criterios para juzgar si el resultado de una decisión judicial está justificado, sin entrar a considerar el procedimiento mediante el cual el juez de hecho ha llegado a esa decisión-resultado. En este punto Amaya ve una ventaja de la teoría coherentista respecto de cualquier otra teoría meramente formal o instrumental. El tipo de justificación al que estas últimas se refieren es siempre relativa y condicionada a la previa aceptación de determinados contenidos que se asumen como empíricamente verdaderos, o normativamente correctos, según sea el caso. A diferencia de ellas, la teoría coherentista no sólo permitiría evaluar si una hipótesis está instrumentalmente justificada, es decir, si es un medio adecuado con relación a un fin, también permitiría identificar cuáles son fines en sí mismos justificados, i.e. hipótesis no instrumental sino intrínsecamente justificada ${ }^{11}$.

Esta tesis es problemática y da lugar a distintas inquietudes. Mencionaré sólo a una. La concepción de Amaya parece suponer algo que antes habíamos descartado. Recuérdese que para la autora, la teoría coherentista es una teoría que permite identificar cuándo ciertos contenidos han de considerase justificados, pero no garantiza que ellos sean verdaderos. Sin embargo, ahora nos dice que, por ejemplo, en el terreno normativo el coherentismo permite identificar no sólo qué pautas estarían instrumentalmente justificadas con relación a un fin, sino también normas en sí mismas justificadas. ¿Qué significa decir que en un terreno normativo la aplicación de un método coherentista no garantiza que lleguemos a contenidos verdaderos pero sí permite identificar fines en sí mismos justificados? ¿Qué diferencia existe entre un contenido normativo en sí mismo justificado y un contenido normativo verdadero? Cabe dudar de que sea posible distinguir estas dos cosas. La sospecha es que cualquier intento de establecer una distinción sería artificial y probablemente ad hoc: inspirado sólo en la necesidad de seguir sosteniendo que la teoría coherentista no permite identificar la verdad, sino sólo aquello que está justificado creer. La

\footnotetext{
${ }^{11}$ Cf. A. Amaya, "Diez tesis acerca de la coherencia en el derecho", apartado 6. En este punto la autora sostiene que el coherentismo ofrece un modelo de justificación no instrumental que permite evaluar fines.
}

76 - Revista Discusiones X 
necesidad de explicar qué significa que la teoría coherentista ofrece un modelo de justificación de fines (un tipo de justificación no relativa ni instrumental) se acentúa aún más si recordamos que la justificación coherentista es siempre contextual. Si la identificación de fines en sí mismos justificados es, como parece, indistinguible de la identificación de contenidos normativos correctos o verdaderos, entonces iel coherentismo permite identificar distintas verdades relativas a distintos contextos?

En segundo lugar, la teoría coherentista de Amaya también compite con todas aquellas otras teorías coherentistas diferentes de la suya. Por ejemplo, compite con las dos propuestas que Amaya señala como buenos ejemplos de teorías coherentistas: las de Richardson y Susan Hurley. Estas últimas son teorías (de justificación epistémica) procedimentales, mientras que la de Amaya ofrece un estándar que evalúa un resultado, independientemente del procedimiento por el que se haya llegado a él. Consecuentemente, lo que puede estar justificado según el estándar propuesto por Amaya, podría no estarlo si aplicamos los procedimientos argumentales sugeridos por Richardson o Hurley. O viceversa, es posible que siguiendo los procedimientos argumentativos de estos autores justifiquemos resultados que no lo estarían conforme al test contrafáctico que es propuesto por Amaya. No es extraño que dentro de un mismo enfoque coherentista de la justificación existan múltiples concepciones, incluso parcialmente incompatibles entre sí. Lo que es extraño, si son incompatibles, es que se defiendan contemporáneamente. En suma, el problema en este caso es el noto predicamento en el que nos encontraremos siempre que asumamos como buenos ciertos procedimientos de toma de decisión (como el respeto de las restricciones negativas y positivas que propone Amaya, o como los que sugieren Hurley o Richardson) y, contemporáneamente, propongamos ciertos criterios que indican resultados correctos (como el criterio contrafáctico de Amaya).

Como sabemos, la tensión entre normas procedimentales y de resultado es especialmente problemática. Si asumimos que no existen, o que no sabemos cuáles serían, resultados justificados, no hay ningún problema en admitir la bondad de uno o más procedimientos de decisión (aun cuando ellos conduzcan a resultados diferentes). Sin embargo, si contamos ya con un 
criterio que identifica los resultados correctos, ¿qué relevancia tienen los procedimientos por los que se llega a dichos resultados? Una vez que tenemos un criterio para juzgar el resultado, o bien los procedimientos carecen de valor y habrán de ser descartados por irrelevantes, o bien tienen valor, en cuyo caso llegaremos inevitablemente a casos de auténtico conflicto entre la exigencia de honrar las pautas procedimentales y la de respetar la pauta que exige un resultado. A tenor de lo dicho en el trabajo de Amaya no es claro qué deberíamos hacer en estos casos de conflicto.

Una última consideración respecto de este tema. Hasta aquí no he mencionado la distinción entre descripción y prescripción, dando por admitido que la teoría coherentista de Amaya persigue un doble propósito explicativo-normativo. Esto es, pretende dar cuenta del modo en el que se llega a creencias justificadas, y pretende también ofrecer un modelo normativo al respecto. Sin embargo, no es banal insistir en la diferencia entre aquellas empresas cuyo objetivo es dar cuenta del modo en que efectivamente se justifica o del modo en que efectivamente se entiende que una decisión está justificada en una práctica real (una práctica jurídica en nuestro caso), y aquellas que proponen una concepción normativa acerca de cómo debería ser, o como sería recomendable que la justificación se realizase o se entendiese.

La pregunta en este contexto es si el coherentismo da efectivamente cuenta del modo en que los jueces justifican sus decisiones (sobre la quaestio iuris y la quaestio facti), o del modo en que la justificación de tales decisiones se concibe o se entiende en la práctica jurídica. También desde este punto de vista, Amaya cree que la teoría coherentista está en ventaja respecto de sus competitivas puesto que, en su opinión, el coherentismo de hecho tiene capacidad descriptiva respecto de nuestras prácticas de justificación ${ }^{12}$.

A pesar de esta opinión, por cuanto se refiere a la justificación judicial de la decisión sobre los hechos y el derecho, un simple test parece mostrar lo contrario. Por ejemplo, aplicando reglas de la deducción, las partes intervinientes en un proceso podrían recurrir la sentencia argumentando que la conclusión a la que arriba el juez no

12 Por ejemplo, según Amaya, la contextualización de los estándares coherentistas "aumenta la adecuación descriptiva de la teoría". Asimismo, 
puede inferirse a partir de las premisas citadas, o que se apoya en un discurso contradictorio. Es decir, podrían impugnar la decisión por falta de justificación en sentido lógico-deductivo, y ello implica que la concepción deductivista está dando cuenta de un aspecto realmente importante acerca de cómo la práctica concibe la justificación de la decisión judicial. Asimismo, las partes podrían impugnar las conclusiones sobre la quaestio facti mostrando que no hay ningún buen argumento inductivo que conduzca a ellas. Nuevamente, ello supone que los argumentos inductivos juegan un rol fundamental en la manera en que, de hecho, la práctica entiende que el juez ha de justificar su decisión sobre los hechos. En tal sentido, el test al que me refiero es el siguiente: ¿podrían las partes en un proceso impugnar las conclusiones del juez demostrando que ellas no son el resultado de un proceso de abducción? $\mathrm{O}$, ¿podrían hacerlo sobre la base de que los jueces no respetan las restricciones (procedimentales o de resultado) que menciona Amaya? La respuesta a estas preguntas es a primera vista negativa. Y ello es indicativo de que, en la práctica jurídica, las decisiones justificadas no se entienden como el coherentismo propone. En todo caso, no es claro cuáles son los datos que demuestran que los operadores jurídicos, cuando ofrecen, critican o reivindican una justificación, la entienden en el modo en que el coherentismo lo hace.

\section{IV}

Me detendré en lo que sigue en dos inquietudes relacionadas con la noción de coherencia que utiliza Amaya en el trabajo que estoy comentando. Como es sabido, hay múltiples problemas relacionados con el concepto de coherencia, y Amaya los ha analizado con profunda atención ${ }^{13}$. Mi primera pregunta en este caso se refiere específicamente

sostiene que es preferible perder precisión "en aras de tener una teoría de la justificación ... que tenga capacidad de describir de manera más fiel nuestras prácticas de justificación" Cf. A. Amaya, "Diez tesis acerca de la coherencia en el derecho", apartado 5 in fine.

${ }^{13}$ Cf. A. Amaya, Tesis doctoral, Cap. 1, punto 4, “Objections Against Coherence Theories of Legal Reasoning". 
a los distintos tipos de relación a los que alude la noción de coherencia y a los distintos objetos de los que la coherencia se predica.

Es claro que en este trabajo, aun cuando la coherencia se predica como propiedad de un conjunto de proposiciones, dicha propiedad es aplicable sólo si existe una determinada relación entre las proposiciones que forman el conjunto. Pero esta relación de coherencia entre proposiciones puede ser entendida como una relación horizontal o vertical ${ }^{14}$. La coherencia horizontal es una relación simétrica de apoyo mutuo entre proposiciones que, por tal razón, se encuentran en un mismo nivel. La coherencia vertical, en cambio, es una relación asimétrica que se establece sólo en una dirección, entre una o más proposiciones y aquellas en las cuales las primeras se apoyan. Amaya, por una parte, está interesada fundamentalmente en la coherencia explicativa de un discurso, que consiste en una relación simétrica existente entre las proposiciones que lo conforman. Pero, por otra parte, habla per-manentemente de la coherencia como de una relación de inferencia, es decir como una relación asimétrica y vertical. Concretamente, como vimos, está muy interesada en sostener que la así llamada "inferencia coherentista" tiene la forma de una inferencia a la mejor explicación.

Una de las conocidas dificultades que se presentan con la justificación coherentista que entiende en términos de una relación (asimétrica) de inferencia es la siguiente. Supongamos que una determinada interpretación del derecho, o una determinada conclusión sobre los hechos, están justificadas por ser las más coherentes respecto de ciertos datos previamente aceptados como relevantes. Ahora bien, ¿cómo sabemos si la aceptación de estos datos está a su vez justificada? Debemos poder concluir que ellos son los más coherentes respecto de un ulterior conjunto de datos, más primario. Pero, ¿cómo determinamos si estos últimos están justificados? Ciertamente una teoría coherentista debe entender la justificación siempre en los mismos términos, y no puede responder que en última instancia nuestro conocimiento se apoya en proposiciones en sí mismas justificadas (auto-evidentes), o que se

\footnotetext{
${ }^{14}$ Cf. J. Raz, "The Relevance of Coherence" in J. Raz, Ethics in The Public Domain, Oxford University Press, Oxford (277-325), pp. 280-2.
} 
justifican mediante un contraste directo con una realidad objetiva. Ello significaría admitir que, en última instancia, la concepción coherentista de justificación fracasa.

Una tesis generalmente aceptada, y que en principio evita este problema, consiste en advertir que la relación de coherencia no pende en última instancia de la justificación de un conjunto final de proposiciones de base porque, en realidad, no es una relación de inferencia asimétrica sino una relación circular. Y si el círculo es suficientemente amplio y robusto no hay por qué pensar que es vicioso ${ }^{15}$. En este punto se pone de manifiesto uno de los aspectos más débiles de la teoría coherentista. Como sabemos, acecha siempre la duda de que el círculo en cuestión, aun cuando sea de gran amplitud y solidez, sea también una coherente ilusión ${ }^{16}$. Una larga y bien tramada novela, perfectamente coherente, no es por ello más probablemente verdadera. Además, esto supone que la relación de coherencia es siempre horizontal y simétrica. Es decir, no hay dos tipos de coherencia, y aunque sea tentador hablar de una "inferencia coherentista", esta expresión es engañosa, la coherencia no es una relación inferencial (i.e. vertical). No es mi objetivo aquí analizar este serio problema que, por otra parte, no es una dificultad de la teoría de Amaya sino del coherentismo en general. En pocas palabras, mi primera inquietud a este respecto es sólo que no se ve del todo claramente cómo debemos entender la noción de coherencia según Amaya. ¿Coherencia es la relación asimétrica que se establece mediante un específico tipo de inferencia: la inferencia coherentista? ¿O es en cambio la relación simétrica que se establece entre dos proposiciones cuando entre ellas hay relaciones recíprocas de inferencia que sólo pueden ser deductivas o inductivas? El problema es que si "coherencia" significa ambas cosas, la expresión se está usando permanentemente en modo ambiguo.

${ }^{15}$ Cf. M. Iglesias, El problema de la discreción judicial. Una aproximación al conocimiento jurídico, Centro de Estudios Políticos y Constitucionales, Madrid, 1999, pp. 211-218.

${ }^{16}$ Según Rescher una teoría coherentista, entre otras exigencias, debe dar una respuesta satisfactoria a la pregunta ¿coherencia respecto de qué? Y lo debería hacer sin confiar en precedentes proposiciones ya establecidas como verdaderas. Cf. N. Rescher, The Coherence Theory of Truth, University Press of America, Washington, 1982, p. 71. 
Esta inquietud está relacionada con otra, no menor. En la propuesta de Amaya, ¿un conjunto de proposiciones o de hipótesis óptimamente coherente lo es con respecto a qué? $\mathrm{O}$, en otros términos, ¿cómo se identifican los datos en que se asientan las conclusiones más coherentes? Amaya reconoce que ${ }^{17}$ :

La teoría de la coherencia como satisfacción de criterios no aborda el tema (esencial) de cómo se genera el conjunto inicial de elementos que se considera relevante para comparar y evaluar la coherencia de las distintas alternativas (el llamado problema del input).

Si es así, resulta difícil compartir la creencia en que esta teoría da cuenta de una relación de justificación más sustancial o más robusta que la justificación inductiva, o menos condicional que la ofrecida por una teoría instrumentalista. Sobre todo, si el problema del input no cuenta con una solución satisfactoria, no se comprende en qué sentido una teoría de este tipo pueda formular la pretensión de estar en condiciones de evaluar si ciertos contenidos son o no fines adecuados, i.e. contenidos justificados, no como medios con relación a un fin sino como fines últimos.

\section{V}

Como vimos, no es claro si la coherencia es el nombre de una específica relación lógica inferencial o el de una relación superviniente, y predicable cuando entre las proposiciones involucradas existen relaciones recíprocas de inferencia deductiva o inductiva. No obstante, es claro que Amaya no pretende sostener que la coherencia es una característica de los sistemas jurídicos. La coherencia es una característica que han de tener las decisiones de los jueces sobre los hechos y el derecho. Según la autora, una teoría coherentista aplicada al

${ }^{17}$ Cf. A. Amaya, "Diez tesis acerca de la coherencia en el derecho", apartado 1 in fine. 
razonamiento judicial es totalmente compatible con una teoría del derecho que reconozca su carácter pluralista y potencialmente conflictivo. En tal sentido, Amaya afirma ${ }^{18}$ :

La coherencia no elimina el conflicto sino que nos proporciona una manera de proceder frente al conflicto. En este sentido, los métodos coherentistas son una herramienta fundamental para realizar la función, primordial, del Derecho, de resolver el conflicto a través de medios argumentativos.

Si admitimos que el derecho protege una multiplicidad de valores de igual jerarquía estamos admitiendo también que a partir del derecho se pueden obtener respuestas incoherentes acerca de lo que se debe hacer en todos aquellos casos individuales en que los conflictos potenciales se actualizan. Justamente, según Amaya, en estos casos en los que el derecho ofrece respuestas en tensión el método coherentista ayudaría a resolver el conflicto, sin necesidad de negarlo.

Esta posición, sin embargo, tiene consecuencias llamativas que ponen en duda la posibilidad de sostener contemporáneamente una teoría coherentista de la justificación de las decisiones judiciales y una teoría no coherentista del derecho. Al respecto pueden señalarse al menos dos tipos de resultados desconcertantes. En primer lugar, en los casos de conflicto entre valores o bienes de igual jerarquía es siempre posible hacer coherente el sistema en más de un sentido. Es decir, por hipótesis, el juez encontrará más de una respuesta igualmente justificada o coherente con el sistema, puesto que éste no es coherente. Parece ser que, si no agregamos a la coherencia algo más, dado el carácter pluralista y potencialmente conflictivo de los derechos contemporáneos, un método coherentista por sí sólo no sirve para determinar una respuesta correcta. No sirve al juez para justificar su decisión, que sólo puede ser una.

En segundo lugar, se podría sostener que la precedente consideración es incorrecta, por incompleta. Es cierto que el método de

${ }^{18}$ Cf. A. Amaya, "Diez tesis acerca de la coherencia en el derecho", apartado 6 in fine. 
justificación coherentista no presupone que los sistemas jurídicos sean coherentes, pero sí presupone que debemos operar bajo el ideal de la coherencia. En tal sentido, en efecto, bajo la presuposición del ideal de un derecho sin conflictos, podemos admitir que el método coherentista es efectivamente la vía mediante la cual reconstruimos el material incoherente convirtiéndolo en un sistema jurídico coherente, que sí permite identificar respuestas correctas. Sin embargo, esto nos conduce a una conclusión un tanto paradójica. Aparentemente, comenzamos reconociendo un valor o un bien en el hecho de que nuestros Derechos sean sistemas pluralistas y protejan distintos derechos -potencialmente conflictivos- confiriéndoles igual dignidad. Sin embargo, terminamos diciendo que debemos operar bajo un ideal no pluralista, que puede ser entendido en dos sentidos diferentes. $\mathrm{O}$ bien como el ideal conforme al cual el derecho es un sistema monista, en el que todos los derechos y obligaciones son instrumentales respecto de un único valor fundamental. O bien como el ideal conforme al cual el derecho está en manos de jueces, decisores hiper-racionales, capaces de precisar el contenido de todos los derechos protegidos en modo tal que ellos no generen conflictos.

Esta segunda opción, aunque extremadamente compleja, parece aún compatible con el valor del pluralismo entendido en términos cuantitativos. Es decir, como el reconocimiento de más de un valor en la más alta posición jerárquica del sistema jurídico. Por ejemplo, en la medida en que los jueces puedan reconstruir con precisión los límites de nuestros conceptos de libertad e igualdad, podríamos sostener que el Derecho protege de igual modo estos dos bienes, contemporáneamente, y que no hay conflictos entre ellos ${ }^{19}$. Sin embargo, se presenta un problema importante. Supongamos que es correcto afirmar que una vez reconstruidos coherentemente los conceptos relevantes por parte del juez el sistema queda determinado: sabemos qué protege cada derecho y evitamos el conflicto entre ellos. El problema es que, en esta hipótesis, es probable que nos hayamos alejado profundamente de lo

\footnotetext{
${ }^{19}$ A mi entender, este es el esfuerzo que la teoría de Dworkin requiere de los jueces que, en tal sentido, deben interpretar para obtener una única respuesta correcta. Cf. R. Dworkin, Law's Empire, Harvard University Press, Cambridge, Mass. 1986. Más específicamente, véase R. Dworkin, “Do Liberal Values
} 
que, en un sentido usual, significa que el derecho es pluralista ${ }^{20}$. Un derecho de este tipo protege no sólo una múltiple cantidad de bienes sino también las múltiples concepciones razonables que de ellos existen en la sociedad. En otras palabras, un sistema jurídico entendido como un sistema debidamente precisado por jueces racionales reconstructores de conceptos coherentes ciertamente satisface el ideal del coherentismo y protege una cantidad múltiple de valores compatibles entre sí. Sin embargo, paradójicamente, dejaría de ser un sistema que respeta los distintos valores tal como ellos se presentan en una sociedad pluralista, tal como en ella se entienden y se pretenden proteger.

Desde un punto de vista moral, ¿es mejor que el derecho proteja un conjunto coherente de valores que proteja distintas concepciones potencialmente conflictivas? Es decir, es mejor que proteja un único modo de entenderlos y no las distintas concepciones existentes en una sociedad plural? ¿Por qué hemos de abrazar un ideal de coherencia si los sistemas jurídicos contemporáneos, por el contrario, reflejan un ideal pluralista? Amaya responde a estas preguntas señalando tres grupos de razones: razones epistémicas, de naturaleza práctica y constitutivas. Entre estas razones, sólo las primeras (que avalan el valor epistémico de la coherencia) hacen referencia a la coherencia entendida como método de conocimiento o teoría epistemológica. El segundo y el tercer grupo de razones se refieren al valor que tiene el contar con un Derecho o conjunto de normas coherentes. Concretamente, por ejemplo, al destacar las razones de naturaleza práctica, Amaya señala que la coherencia promueve la seguridad y eficacia jurídicas, ya que es más fácil comprender y recordar, aplicar y seguir un conjunto de normas coherentes que una serie de normas inconexas ${ }^{21}$.

Esto parece avalar la impresión de que, si no imposible, es difícil sostener que la coherencia es un valor aplicable a las decisiones

Conflict?", en Mark Lilla, Ronald Dworkin, and Robert B. Silvers (eds.), The Legacy of Isaiah Berlin, New York Review of Books, New York, 2002, pp. 73-90.

${ }^{20}$ Cf. B. Williams, "Liberalism and Loss", en Mark Lilla, Ronald Dworkin, and Robert B. Silvers (eds.), op.cit. pp. 91-104.

${ }^{21}$ Cf. A. Amaya, "Diez tesis acerca de la coherencia en el derecho", apartado 7. 
judiciales sin sostener contemporáneamente que es un valor aplicable al Derecho en sentido objetivo: a los sistemas jurídicos. Pero, si esto es así, si sostenemos el valor de la coherencia de los sistemas jurídicos, es desconcertante sostener al mismo tiempo el valor del pluralismo. Admitir el valor del pluralismo, en un sentido no meramente cuantitativo, implica aceptar como un dato no problemático la presencia de conflictos y dar la bienvenida a la existencia de múltiples respuestas (aunque no necesariamente cualquier respuesta) igualmente correctas. Ello es así puesto que se acepta como dato positivo el hecho de que haya una variedad de concepciones respetables de un mismo valor. Por el contrario, defender el valor de la coherencia presupone aceptar que el Derecho, aun cuando reconozca y proteja una pluralidad de bienes, en realidad protege una única concepción de los mismos: "la" concepción correcta de cada uno de ellos. Esto, lejos de dar la bienvenida a los distintos puntos de vista presentes en una sociedad plural implica concebirlos como una dificultad que es preciso superar. Y está claro que, en este caso, serían los jueces los encargados de identificar "la" concepción correcta de los mismos sobre la base de una teoría epistemológica coherentista.

No cabe duda alguna que, conforme a las opiniones de Amaya, la coherencia es un valor. Deberíamos decir, quizás, un meta-valor que requiere la reconstrucción armoniosa de los distintos valores o bienes protegidos por el derecho. No es claro, sin embargo, si también lo es el pluralismo. Valor que reside, no sólo en que los sistemas jurídicos protejan una cantidad plural de bienes, sino en que los protejan conforme a las distintas concepciones razonables que en la sociedad existen de los mismos, y que probablemente no son coherentes entre sí. 